\title{
Cyclostationary Neural Networks for Air Pollutant Concentration Prediction
}

\author{
Monica Bianchini, Ernesto Di Iorio, Marco Maggini, and Augusto Pucci \\ Dipartimento di Ingegneria dell'Informazione \\ Via Roma 56, I-53100 Siena (Italy) \\ \{monica, diiorio, maggini, augusto\}@dii.unisi.it
}

\begin{abstract}
There are many substances in the air which may impair the health of plants and animals, including humans, that arise both from natural processes and human activity. Nitrogen dioxide $\mathrm{NO}_{2}$ and particulate matter $\left(\mathrm{PM}_{10}, \mathrm{PM}_{2.5}\right)$ emissions constitute a major concern in urban areas pollution. The state of the air is, in fact, an important factor in the quality of life in the cities, since it affects the health of the community and directly influences the sustainability of our lifestyles and production methods. In this paper we propose a cyclostationary neural network (CNN) model for the prediction of the $\mathrm{NO}_{2}$ and $\mathrm{PM}_{10}$ concentrations. The cyclostationary nature of the problem guides the construction of the CNN architecture, which is composed by a number of MLP blocks equal to the cyclostationary period in the analyzed phenomenon, and is independent from exogenous inputs. Some experiments are also reported in order to show how the CNN model significantly outperforms standard statistical tools and linear regressors usually employed in these tasks.
\end{abstract}

\section{Introduction}

There are many substances in the air which may impair the health of plants and animals, including humans, that arise both from natural processes and human activity. Substances not naturally found in the air, or at greater concentrations, or in different locations from usual, are referred to as pollutants. Pollutants can be classified as either primary or secondary. Primary pollutants are substances directly produced by a process, such as ash from a volcanic eruption or the carbon monoxide gas from a motor vehicle exhaust. Instead, secondary pollutants are not emitted. Rather, they form in the air when primary pollutants react or interact. An important example of a secondary pollutant is ozone - one of the many secondary pollutants that constitute the photochemical smog. Note that some pollutants may be both primary and secondary: that is, they are both emitted directly and formed as combinations of other primary pollutants. Primary pollutants produced by human activity include:

- Oxides of sulfur, nitrogen and carbon;

- Organic compounds, such as hydrocarbons (fuel vapour and solvents);

- Particulate matter, such as smoke and dust;

L. Prevost, S. Marinai, and F. Schwenker (Eds.): ANNPR 2008, LNAI 5064, pp. 101-112, 2008.

(C) Springer-Verlag Berlin Heidelberg 2008 
- Metal oxides, especially those of lead, cadmium, copper and iron;

- Toxic substances.

Secondary pollutants include some particles formed from gaseous primary pollutants and compounds in the photochemical smog, such as nitrogen dioxide, ozone and peroxyacetyl nitrate (PAN).

The main oxides of nitrogen present in the atmosphere are nitric oxide (NO), nitrogen dioxide $\left(\mathrm{NO}_{2}\right)$ and nitrous oxide $\left(\mathrm{N}_{2} \mathrm{O}\right)$. Nitrous oxide occurs in much smaller quantities than the other two, but it is of interest as it represents a powerful greenhouse gas and thus contributes to global warming. The major human activity which generates oxides of nitrogen is fuel combustion, especially in motor vehicles. Oxides of nitrogen form in the air when fuel is burnt at high temperatures. This is mostly in the form of nitric oxide with usually less than $10 \%$ as nitrogen dioxide. Once emitted, nitric oxide combines with ozone $\left(\mathrm{O}_{3}\right)$ to form nitrogen dioxide, especially in warm sunny conditions. These oxides of nitrogen may remain in the atmosphere for several days and, during this time, chemical processes may generate nitric acid, and nitrates and nitrites as particles. The oxides of nitrogen play a major role in the chemical reactions which generate the photochemical smog. Nitrogen dioxide is also a respiratory irritant which may worsen the symptoms of an existing respiratory illness.

On the other hand, particulate matter $\left(\mathrm{PM}_{10}\right)$ pollution consists of very small liquid and solid particles floating in the air. Sources of $\mathrm{PM}_{10}$ in both urban and rural areas are motor vehicles, wood burning stoves and fireplaces, dust from construction, landfills, and agriculture, wildfires and waste burning, industrial sources, windblown dust from open lands. In particular, of greatest concern to public health, are the $\mathrm{PM}_{10}$ particles small enough to be inhaled into the deepest parts of the lung [16[19]20. These particles are less than 10 microns in diameter, and result from a mixture of materials that can include smoke, soot, dust, salt, acids, and metals.

Many modelling efforts have been recently spent for controlling the $\mathrm{NO}_{2}$ and $\mathrm{PM}_{10}$ concentrations in order to enable the development of tools for pollution management and reduction. One approach to predict future pollutant concentrations is to use detailed atmospheric diffusion models (see [2, for a review). Such models aim at solving the underlying physical and chemical equations that control pollutant concentrations and, therefore, require clean emission data and meteorological fields. An alternative approach is to devise statistical models which attempt to determine the underlying relationship between a set of input data and targets. Regression modelling is an example of such a statistical approach and has been applied to air quality modelling and prediction in a number of studies [72122]. One of the limitations imposed by linear regression tools is that they will underperform when used to model non-linear systems. Instead, artificial neural networks can model non-linear systems and have been succesfully used for predicting air pollution concentrations (see, f.i., [5|6|9|11|12|13|14|15|18]).

In this paper, we propose a cyclostationary neural network $(\mathrm{CNN})$ architecture to model and estimate hourly the $\mathrm{NO}_{2}$ concetrations, and to obtain a 1-day ahead prediction for the $\mathrm{PM}_{10}$. The cyclostationary nature of the problem guides 
the construction of the CNN, which is composed by a number of MLP blocks equal to the duration of the cyclostationary period in the analyzed phenomenon, specifically 24 hours for the prediction of the $\mathrm{NO}_{2}$ concentration and a week (7 days) for the $\mathrm{PM}_{10}$. The novelty of our approach particularly lies on its independence from exogenous data, in that it uses only the time series of $\mathrm{NO}, \mathrm{NO}_{2}$, and $\mathrm{PM}_{10}$, respectively, to predict their future values. In fact, meteorological data are not taken (explicitly) into account, suggesting that the network is able to detect the necessary exogenous information directly from the pollution data. Therefore, the proposed CNN architecture is robust w.r.t. geographical and seasonal changes. Some experimentation was carried out on the data gathered by ARPA (Agenzia Regionale per la Protezione dell'Ambiente — Regional Environmental Protection Agency) of Lombardia (northern Italy). ARPA supplies a real-time air quality monitoring system to defend the people health and the region ecosystem quality. Experimental results are very promising and show that the CNN model significantly outperforms standard statistical tools — like AutoRegressive eXogenous (ARX) models — and linear regressors, usually employed for this task [4.

The paper is organized as follows. In the next section, the CNN architecture is introduced and the data preprocessing, aimed at creating a learning set tailored to the CNN model, is reported. Section 3 describes the experimental setup, respectively for the nitrogen dioxide and the particulate matter concentrations, comparing the performance of the proposed method with AR models and linear regression tools. Finally, Section 4 reports the conclusions.

\section{Cyclostationary Neural Networks}

A discrete time random process $X(t)$ is a rule for assigning to every outcome of an experiment $\zeta$ a function $X(t, \zeta)$. The domain of $\zeta$ is the set of all the experimental outcomes and the domain of $t$ is a set of natural numbers [17. Thus, a random process is a set of random variables, one for each time instant $t$. If the statistics of a random process change over time, then the process is called nonstationary. The subclass of nonstationary processes whose statistics vary periodically with time are called cyclostationary.

Whenever the cyclostationarity period $T$ is known, a set of $T$ stationary processes can be derived from the original one [10], on which different neural networks can be trained independently to predict the outcomes of the related random variables. Therefore, the CNN consists of a set of $T$ independent but with an identical architecture - MLPs, each modelling a random variable of the original cyclostationary process. Formally speaking, for a cyclostationary process $X$ with period $T$, the set of all the outcomes $A^{*}=\left\{a_{j} \mid j \in[0, \infty)\right\}$ can be partitioned into $T$ subsets, one for each random variable, that is $A^{*}=$ $\left\{A_{0}, A_{2}, \ldots, A_{T-1}\right\}$, where $A_{i}=\left\{a_{j} \mid i=j \bmod T\right\}$. The $i-t h$ MLP will be trained on the subset of the outcomes concerning the $i$-th random variable of the process. 


\subsection{Prediction of the $\mathrm{NO}_{2}$ Concentration}

This prediction task consists in modeling the $\mathrm{NO}_{2}$ time series, based on the past concentrations of $\mathrm{NO}$ and $\mathrm{NO}_{2}$. In this case, it is easily verifiable that a strong correlation exists between the past $\mathrm{NO}$ data and the current value of the $\mathrm{NO}_{2}$ concentration, with a periodicity of 24 hours. This means that the $\mathrm{NO}_{2}$ pollution at time $t$ depends on the NO sampled at $t-24, t-48$, etc. Therefore, we claim that the process we are analyzing has a cyclostationary period $T=24$, i.e. a daily periodicity, and, consequently, a CNN model will be composed by 24 MLP blocks. In particular, each MLP - one for each random variable of the cyclostationary process - will exploit $\mathrm{NO}(t-T)$ and $\mathrm{NO}_{2}(t-1)$ to predict $\mathrm{NO}_{2}(t)$. Formally:

$$
\mathrm{NO}_{2}(t)=f_{k}\left(\mathrm{NO}_{2}(t-1), \mathrm{NO}(t-T)\right), \quad k=t \bmod T,
$$

where $T=24$ and $f_{k}, k=0, \ldots, T-1$, represents the $k$-th approximation function realized by the $k$-th MLP block.

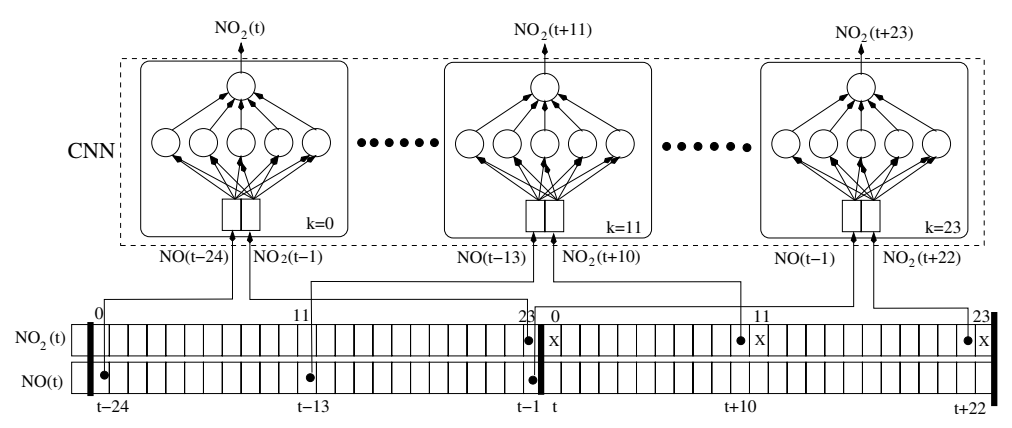

Fig. 1. The CNN architecture and the data sampling procedure

It is worth mentioning that the CNN model relies just on $\mathrm{NO}$ and $\mathrm{NO}_{2}$ time series values. In fact, it is completely independent of exogenous data, such as weather condition (i.e. pressure, wind, humidity, etc.) and geographic conformation. This is just an interesting feature, since we can avoid to predict such weather conditions and focus only on the $\mathrm{NO}_{2}$ concentration prediction. The resulting model will obviously be much more robust against noise and prediction error.

\subsection{Prediction of the $\mathrm{PM}_{10}$ Concentration}

In order to predict the particulate matter pollution, and since $\mathrm{PM}_{10}$ time series underlies clear periodicities at the yearly and weekly level, we consider a CNN neural network composed by 7 MLP blocks to forecast the $\mathrm{PM}_{10}$ daily average concentration. To be used as an input for the predictor, each monitored parameter has to be grouped from the original hourly series to a daily time series; this has been accomplished calculating the average over the whole day. Therefore, the 
$k$-th MLP block calculates the function $f_{k}$, for the 1 -day prediction of $\mathrm{PM}_{10}$, based on the today-value and on the concentration measured a week before:

$$
\mathrm{PM}_{10}(t)=f_{k}\left(\mathrm{PM}_{10}(t-1), \mathrm{PM}_{10}(t-W)\right), \quad k=t \bmod W,
$$

where $W=7$ and $f_{k}, k=0, \ldots, W-1$, represents the $k$-th approximation function realized by the $k$-th MLP block.

As observed for the nitrogen dioxide, even for the case of the particulate matter, the CNN network is able to make the 1-day prediction without taking into account other environmental conditions except for the past concentrations of the pollutant to be predicted.

\section{Experimental Results}

In order to assess the capability of the proposed neural network model to predict cyclostationary phenomena and, in particular, air pollutant concentrations, several experiments have been performed to compare the CNN model to other models both for the prediction of the $\mathrm{NO}_{2}$ hourly concentrations and for the prediction of the daily concentration of the $\mathrm{PM}_{10}$, which is known to be a harder task (see, f.i. 8911) to be faced with connectionist models.

In this work, we used data gathered by the ARPA of Lombardia (northern Italy). ARPA supplies a real-time air quality monitoring system to defend the people health and the region ecosystem quality. The ARPA air quality monitoring system is composed by mobile and fixed stations.

The first dataset is made up by the nitric oxide and dioxide concentrations detected hourly by several stations in Bergamo and Brescia (two important cities in Lombardia) and by the unique station in Breno (a small city close to Brescia) $)^{1}$. Instead, for the particulate matter prediction task, the data are gathered from a monitoring station located in a residential area of Milan. The dataset is constituted by a hourly time series, with a missing value rate ranging between $5 \%$ and $10 \%$.

\subsection{Experiments on the Prediction of the $\mathrm{NO}_{2}$ Concentration}

To test the CNN model for the prediction of the nitrogen dioxide concentration, we exploited 21 different sets of data, gathered from seven monitoring stations, three in Bergamo (Via Garibaldi, Via Goisis, Via Meucci), three in Brescia (Broletto, via Trimplina, Via Turati), and the unique one in Breno. Three different datasets were defined corresponding to the measurements collected during different (or partially overlapped) time periods, as shown in Table 1

For each dataset, the performance of the CNN architecture based on 24 MLPs, as described in Section 2. was compared with a similar architecture based on a set of 24 AutoRegressive eXogenous input (ARX) models. By a trial and error

${ }^{1}$ The dataset and some related information are available at the web site http://www.arpalombardia.it/qaria/doc_RichiestaDati.asp 
Table 1. Datasets used in the experiments

\begin{tabular}{|c|c|c|}
\hline Label & Learning set & Test set \\
\hline $2003-2004 / 2005$ & $\begin{array}{c}1-1-2003 \text { 1:00 a.m. } \\
\text { to } \\
1-1-2005 \text { 0:00 a.m. }\end{array}$ & $\begin{array}{l}1-1-20051: 00 \text { a.m. } \\
\text { to } \\
1-1-2006 \text { 0:00 a.m. }\end{array}$ \\
\hline $2003 / 2004$ & $\begin{array}{c}1-1-2003 \text { 1:00 a.m. } \\
\text { to } \\
1-1-2004 \text { 0:00 a.m. }\end{array}$ & $\begin{array}{l}\text { 1-1-2004 1:00 a.m. } \\
\text { to } \\
\text { 1-1-2005 0:00 a.m. }\end{array}$ \\
\hline $2004 / 2005$ & $\begin{array}{l}1-1-2004 \text { 1:00 a.m. } \\
\text { to } \\
1-1-2005 \text { 0:00 a.m. }\end{array}$ & $\begin{array}{l}1-1-2005 \text { 1:00 a.m. } \\
\text { to } \\
1-1-2006 \text { at 0:00 a.m. }\end{array}$ \\
\hline
\end{tabular}
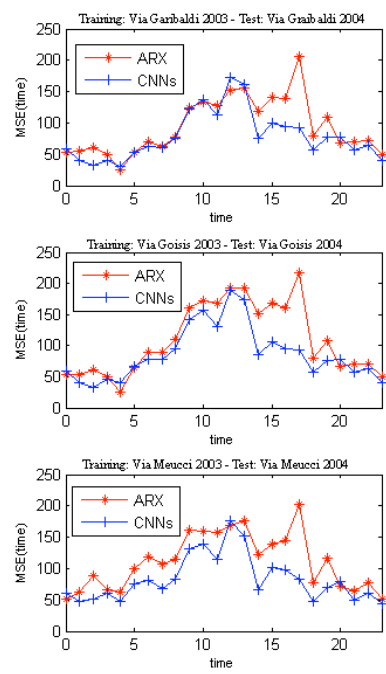
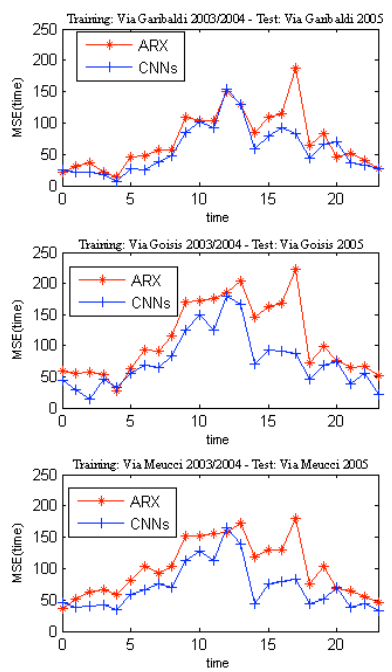
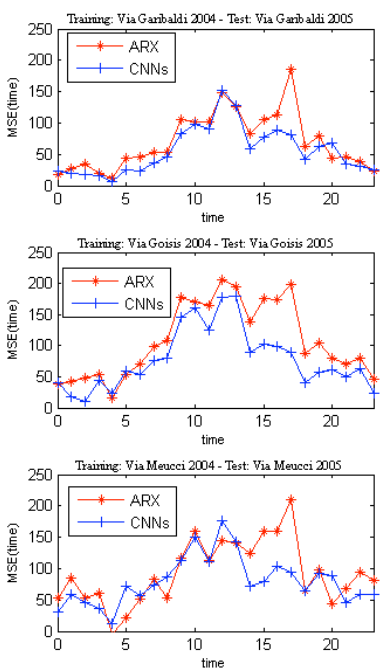

Fig. 2. Average absolute error for the prediction of the $\mathrm{NO}_{2}$ hourly concentration at the three station in Bergamo. Each column corresponds to one of the three datasets of Table 1

procedure, a two-layer neural network architecture with five hidden neurons was chosen for the CNN model. Figure 2 shows the results for the three stations in Bergamo. In each plot, the $\mathrm{x}$-axis corresponds to the time of the day, while the $\mathrm{y}$-axis corresponds to the average value of the absolute prediction error computed for all the days in the test set. The absolute prediction error is defined as $e(t)=|y(t)-\hat{y}(t)|$, where $y(t)$ is the current $\mathrm{NO}_{2}$ value at time $t$ and $\hat{y}(t)$ is the model estimation. By comparing the two error curves, it can be noted that the CNN absolute error is quite often significantly smaller than the error for the ARX model, both with respect to the station and the time of the day. Similar results were obtained for the stations in Brescia and Breno. 

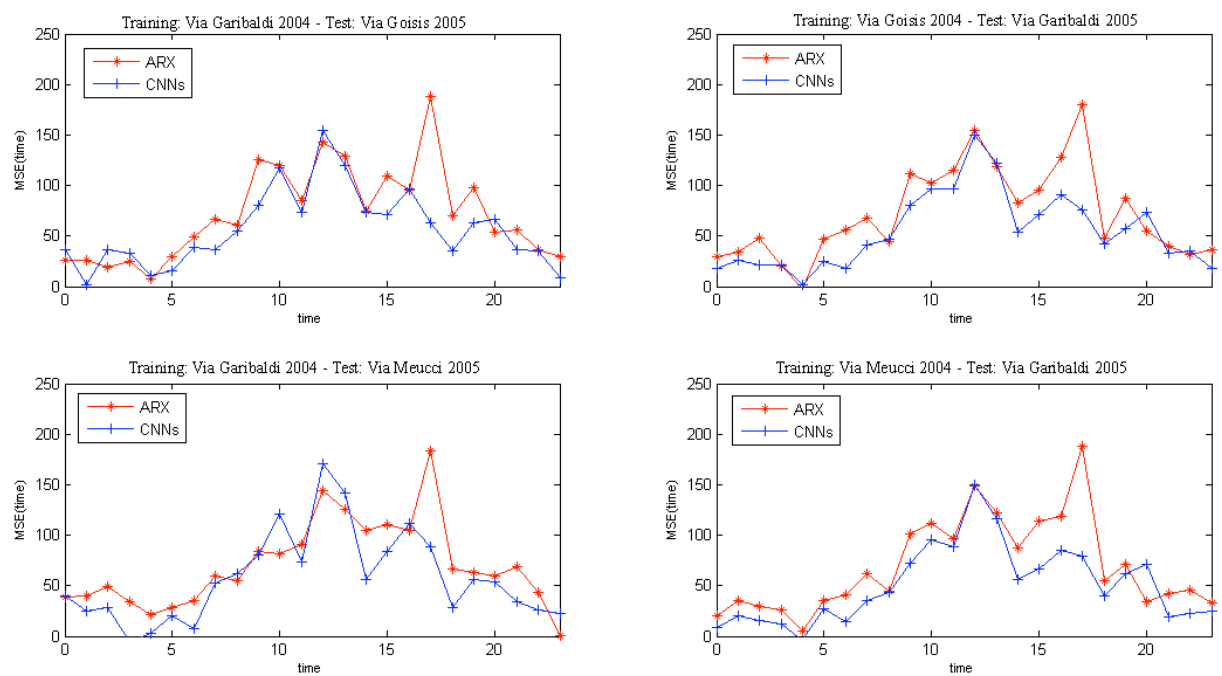

Fig. 3. Experimental results obtained by using the $\mathrm{NO}-\mathrm{NO}_{2}$ hourly concentration of a Bergamo's station to train the CNN, whose performance is then tested based on data gathered from another Bergamo's station.

A second set of experiments was aimed at evaluating the generalization performance of the CNN model with respect to the position of the monitoring station used to train the model and to the station considered for the prediction. Thus, the $\mathrm{CNN}$ was trained by using the $\mathrm{NO}-\mathrm{NO}_{2}$ time series gathered at a specific station and tested on the concentration collected, in the same period, at a different monitoring point. In particular, three different types of experiments were carried out:

- Stations of the same city. For each city, a CNN was trained for each NO$\mathrm{NO}_{2}$ time series and then tested on the data gathered at the other stations of the same city;

- Stations of close cities. A CNN was trained based on the $\mathrm{NO}-\mathrm{NO}_{2}$ time series measured at a certain station of a particular city, and then tested on the data gathered at some stations of a close city (for example, we use the concentration of the station at Brescia - Via Turati for the training and that of the station of Breno for the testing phase);

- Stations of far cities. A CNN was trained based on the $\mathrm{NO}-\mathrm{NO}_{2}$ time series measured at a certain station of a particular city, and then tested on the data gathered at some stations of a far city (e.g., using data from Brescia for the training and from Bergamo for the testing phase).

Figure 3 reports the results when considering four different combinations of the three measuring stations in Bergamo for the 2004/2005 dataset. The plots show that even in this case the CNN model performs better than the ARX 

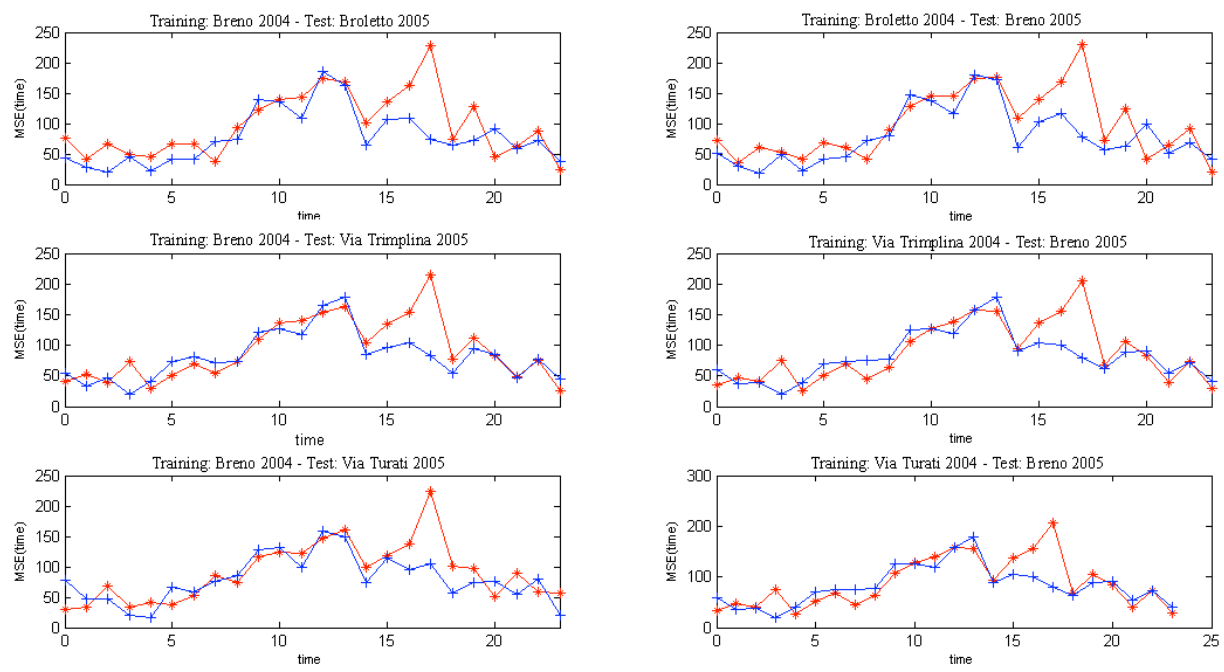

Fig. 4. Experimental results obtained on the 2004/2005 dataset by using the NO- $\mathrm{NO}_{2}$ hourly concentration of the Breno's station for training and data gathered at some Brescia's stations for testing.

model. In particular, it is interesting to note that this experimental setup does not lead to a relevant CNN performance degradation.

In Figure 4, the results obtained by training the CNN on the hourly concentration of the Breno's station and testing it on the Brescia's stations are shown. This is the case of stations in nearby cities, and, as shown in the plots, there is not a significant performance degradation for the CNN model. We obtained similar results also by training the CNN model on a given station in Brescia and testing it on the data of the Breno's station.

In Figure 5] the plots show the average absolute error when the CNN is trained by using the hourly concentration of the Breno's station and then tested on the Bergamo's stations (and vice versa). In this case, the geographic distance among the stations is furthermore increased, nevertheless maintaining the CNN performance almost unchanged.

Finally, we investigated how the size of the learning set affects the prediction accuracy of the proposed model. We considered the 2004/2005 dataset that corresponds to two years of data and we adopted the following scheme. For each week, we considered the previous $w$ weeks with $w=1, \ldots, 25$. Starting from the 26-th week in the dataset, for each value of $w$, we predicted the hourly $\mathrm{NO}_{2}$ concentration for each day, using a CNN model trained on the data collected for the days in the previous $w$ weeks. For each learning set size $w$, we first computed the Mean Square Error for the hourly prediction in each week, and then we furtherly averaged this value on all the weeks in the test set. The results are shown in the plots in Figure 6, where the $\mathrm{x}$-axis is labelled by the parameter $w$ - the number of weeks on which the model was trained — whereas the corresponding 

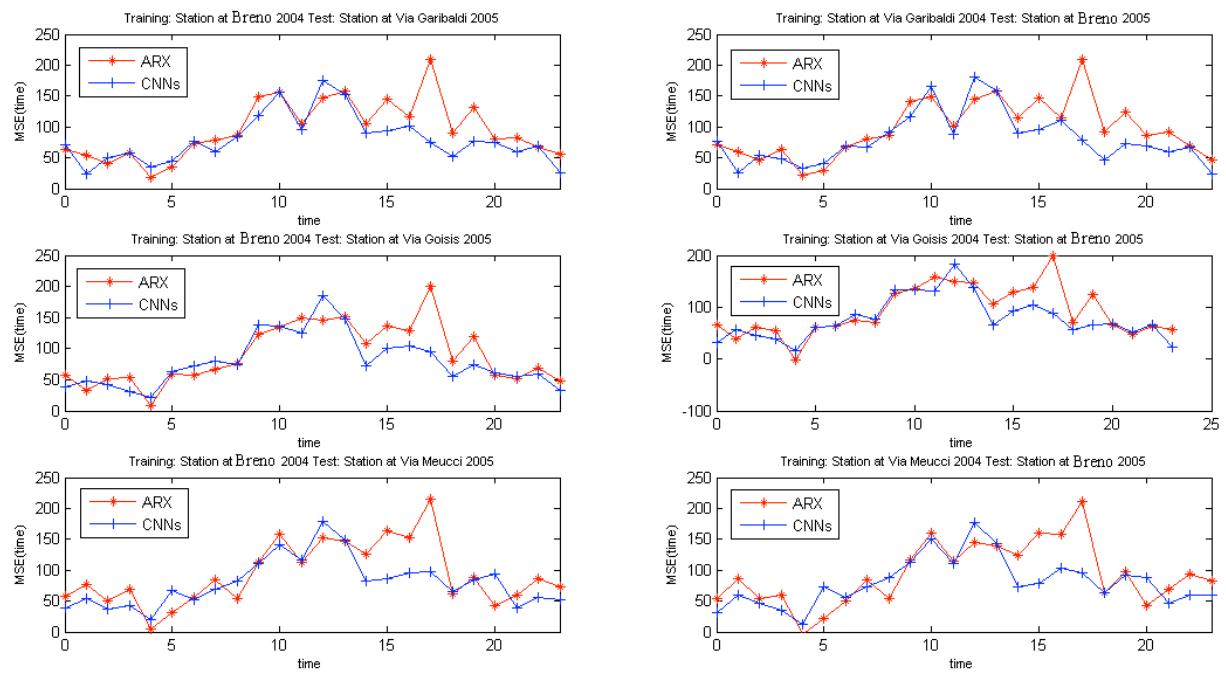

Fig. 5. Experimental results obtained by using the $\mathrm{NO}-\mathrm{NO}_{2}$ hourly concentration of the Breno's station for training and data gathered at some Bergamo's stations for testing and vice versa.

average MSE is reported on the $\mathrm{y}$-axis. The different curves refer to the three stations in Brescia - Figure 6 (a) - and in Bergamo - Figure 6 (b). It is easy to note a common trend in each dataset, that corresponds to a performance improvement when the training window size increases up to 12-14 weeks, whereas there are not very significant changes when $w>14$. Interestingly, this size for $w$ corresponds exactly to an entire season. This result reveals a seasonal periodicity in the considered phenomenon.

\subsection{Experiments on the Prediction of the $\mathrm{PM}_{10}$ Concentration}

The second experimental setup is aimed at presenting a comparison among CNNs, a neural network approach (also based on MLPs) proposed in 1], and a linear predictor described in [3, on the $\mathrm{PM}_{10}$ 1-day ahead prediction. In this case the CNN architecture is based on a set of 7 two-layer MLPs, with 15 hidden neurons each. This architecture was determined by a trial and error procedure and assumes a weekly cyclostationarity period. The dataset is constituted by a hourly time series collected in the period 1999-2002 from a monitoring station located in a residential area of Milan. The data are grouped from the original hourly series into a daily time series and then splitted to form the training set (1999-2000), the validation set (2001), and the test set (2002), respectively.

In 11, many exogenous features are used as inputs for the neural network model to perform the $\mathrm{PM}_{10}$ 1-day ahead prediction, including an autoregressive $\mathrm{PM}_{10}$ term, the past concentrations of $\mathrm{NO}, \mathrm{NO}_{2}$, and $\mathrm{SO}_{2}$, and a wide set of meteorological variables, such as temperature, humidity, wind velocity, solar 


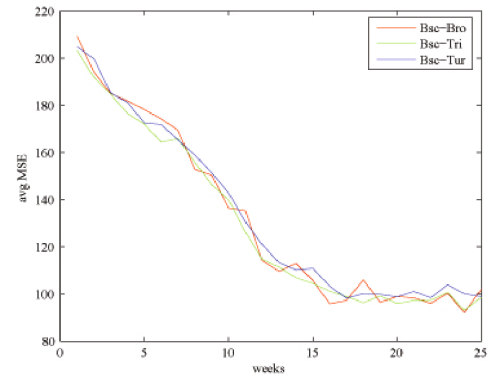

(a)

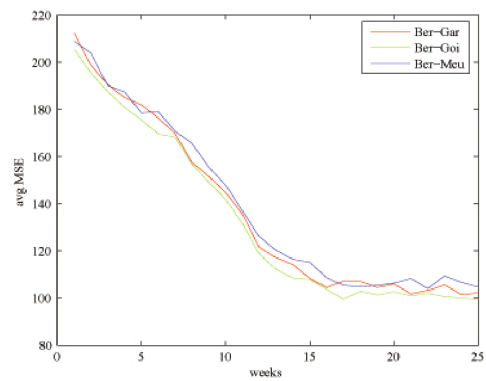

(b)

Fig. 6. Weekly Mean Square Error on the hourly $\mathrm{NO}_{2}$ prediction task with respect to the number $w$ of past weeks in the learning set. (a) Brescia stations. (b) Bergamo stations.

Table 2. Prediction Mean Absolute Error (MAE) on the $\mathrm{PM}_{10}$ 1-day ahead prediction task

\begin{tabular}{|l|l|l|l|}
\hline & CNNs & Cecchetti et al. [1] & Corani et al. [3] \\
\hline MAE & $8.34 \mathrm{mg} / \mathrm{m}^{3}$ & $8.71 \mathrm{mg} / \mathrm{m}^{3}$ & $11 \mathrm{mg} / \mathrm{m}^{3}$ \\
\hline
\end{tabular}

radiation, atmospheric pressure and the Pasquill stability class, a commonly used method of categorizing the amount of atmospheric turbulence 2 . Instead, in our approach, we just exploited the ad hoc past values of the $\mathrm{PM}_{10}$ concetrations (those calculated the day before and one week before).

Table 2 reports the results obatined on the same data by CNNs, and the other approaches respectively proposed in [1] and [3].

\section{Conclusions}

In this paper, a connectionist model called Cyclostationary Neural Network (CNN) was introduced, particularly tailored for the prediction of cyclostationary phenomena. In particular, the CNN architecture was introduced to model and estimate hourly the nitrogen dioxide $\left(\mathrm{NO}_{2}\right)$ concetrations, and to obtain a 1-day ahead prediction for the particulate matter $\left(\mathrm{PM}_{10}\right)$, both pollutants playing a major role in the chemical reactions which generate the photochemical smog. One fundamental peculiarity of the proposed model is that of being independent of evironmental, tipically metereological, exogenous data, in that the $\mathrm{NO}_{2}$ concentration prediction is based only on the previous level of $\mathrm{NO}_{2}$

${ }^{2}$ The six stability classes are named A, B, C, D, E, and F, with class A being the most unstable or most turbulent class, and class $\mathrm{F}$ the most stable or least turbulent class. Each class is determined by the ranges of values for the surface windspeed, the daytime incoming solar radiation, and the nighttime cloud cover. 
and of the nitric oxide $(\mathrm{NO})$ - which combines with ozone $\left(\mathrm{O}_{3}\right)$ to form $\mathrm{NO}_{2}$ whereas the $\mathrm{PM}_{10}$ prediction depends only on the previous values of the same pollutant. Some experimentation, carried out on the data gathered by ARPA Lombardia, looks very promising and shows that the CNN model significantly outperforms standard statistical tools, like ARX models, usually employed for the air pollutant prediction task.

\section{References}

1. Cecchetti, M., Corani, G., Guariso, G.: Artificial neural networks prediction of $\mathrm{PM}_{10}$ in the Milan area. In: $2^{\text {nd }}$ International Environmental Modelling and Software Society Conference (2004)

2. Collet, R.S., Oduyemi, K.: Air quality modelling: A technical review of mathematical approaches. Metereological Applications 4(3), 235-246 (1997)

3. Corani, G., Barazzetta, S.: First results in the prediction of particulate matter in the Milan area. In: $9^{\text {th }}$ Int. Conf. on Harmonisation within Atmospheric Dispersion Modelling for Regulatory Purposes (2004)

4. Finzi, G., Volta, M., Nucifora, A., Nunnari, G.: Real time ozone episode forecast: A comparison between neural network and grey box models. In: Proceedings of International ICSC/IFAC Symposium of Neural Computation, pp. 854-860. ICSC Academic Press, London (1998)

5. Gardner, M.W., Dorling, S.R.: Artificial neural networks (the multilayer perceptron) - A review of applications in the atmospheric sciences. Atmospheric Environment 32(14-15), 2627-2636 (1998)

6. Gardner, M.W., Dorling, S.R.: Neural network modelling and prediction of hourly $\mathrm{NO}_{x}$ and $\mathrm{NO}_{2}$ concentration in urban air in London. Atmospheric Environment 33, 709-719 (1999)

7. Goyal, P., Chanb, A.T., Jaiswa, N.: Statistical models for the prediction of respirable suspended particulate matter in urban cities. Atmospheric Environment 40(11), 2068-2077 (2006)

8. Hooyberghs, J., Mensink, C., Dumont, G., Fierens, F., Brasseur, O.: A neural network forecast for daily average $\mathrm{PM}_{10}$ concentrations in Belgium. Atmospheric Environment 39(18), 3279-3289 (2005)

9. Kukkonen, J., Partanen, L., Karppinen, A., Ruuskanen, J., Junninen, H., Kolehmainen, M., Niska, H., Dorling, S.R., Chatterton, T., Foxall, R., Cawley, G.: Extensive evaluation of neural network models for the prediction of $\mathrm{NO}_{2}$ and $\mathrm{PM}_{10}$ concentrations, compared with a deterministic modelling system and measurements in central Helsinki. Atmospheric Environment 37, 4539-4550 (2003)

10. Ljung, L.: System Identification — Theory for the User, 2nd edn. PTR Prentice Hall, Upple Saddle River (1999)

11. Lu, W.Z., Fan, H.Y., Lo, S.M.: Application of evolutionary neural network method in predicting pollutant levels in downtown area of Hong Kong. Neurocomputing 51, 387-400 (2003)

12. Lu, W.Z., Wang, W.J., Xu, Z.B., Leung, A.Y.: Using improved neural network model to analyze RSP, $\mathrm{NO}_{x}$ and $\mathrm{NO}_{2}$ levels in urban air in Mong Kok, Hong Kong. Environmental Monitoring and Assessment 87(3), 235-254 (2003)

13. Morabito, F.C., Versaci, M.: Wavelet neural network processing of urban air pollution. In: Proceedings of IJCNN 2002, Honolulu (Hawaii), vol. 1, pp. 432-437. IEEE, Los Alamitos (2002) 
14. Nunnari, G., Cannavò, F.: Modified cost functions for modelling air quality time series by using neural networks. In: Kaynak, O., Alpaydın, E., Oja, E., Xu, L. (eds.) ICANN 2003 and ICONIP 2003. LNCS, vol. 2714, pp. 723-728. Springer, Heidelberg (2003)

15. Ordieres, J.B., Vergara, E.P., Capuz, R.S., Salazar, R.E.: Neural network prediction model for fine particulate matter $\left(\mathrm{PM}_{2.5}\right)$ on the US-Mexico border in El Paso (Texas) and Ciudad Juárez (Chihuahua). Environmental Modelling and Software 20(5), 547-559 (2005)

16. Ostro, B., Chestnut, L., Vichit-Vadakan, N., Laixuthai, A.: The impact of particulate matter on daily mortality in Bangkok, Thailand. Journal of Air and Waste Management Association 49, 100-107 (1999)

17. Papoulis, A.: Probability, Random Variables, and Stochastic Processes, 3rd edn. McGraw-Hill, New York (1991)

18. Perez, P., Reyes, J.: Prediction of maximum of $24-\mathrm{h}$ average of $\mathrm{PM}_{10}$ concentrations $30 \mathrm{~h}$ in advance in Santiago, Chile. Atmospheric Environment 36, 4555-4561 (2002)

19. Pope, C.A., Burnett, R., Thun, M.J., Calle, E.E., Krewskik, D., Ito, K., Thurston, G.D.: Lung cancer, cardiopulmonary mortality, and long term exposure to fine particulate air pollution. Journal of the American Medical Association 287, 11321141 (2002)

20. Pope, C.A., Thun, M.J., Namboodiri, M.M., Dockery, D.W., Evans, J.S., Speizer, F.E., Heath, C.W.: Particulate air pollution as predictor of mortality in a prospective study of US adults. American Journal of Respiratory and Critical Care Medicine 151, 669-674 (1995)

21. Wang, J.Y., Lord, E., Cannon, A., Walters, G.: Statistical models for spot air quality forecasts $\left(\mathrm{O}_{3}\right.$ and $\left.\mathrm{PM}_{10}\right)$ in British Columbia. In: Proceedings of the 2005 Puget Sound Georgia Basin Research Conference, Seattle (2005)

22. Zolghadri, A., Henry, D.: Minimax statistical models for air pollution time series, Application to ozone time series data measured in Bordeaux. Environmental Monitoring and Assessment 98(1-3), 275-294 (2004) 